\title{
A Study on Multi-body Dynamic Modelling and Optimization for Mechanical Quick Closing Valve
}

\author{
Wang $\mathrm{Bo}^{\mathrm{a}}$, Li Junyan, Wu Lifang and Chen Xuekai \\ Research Institute of Physical and Chemical Engineering of Tianjin, Tianjin 300180, China
}

\begin{abstract}
The multi-body dynamic modelling and optimization methods of mechanical quick closing valve are studied in this paper. The Lagrange equation is specified characterizing the closing process. Normal contact force and tangent friction force between the components are obtained by using equivalent spring-damper model and no-linear coulomb friction model. The theoretical result of the valve's kinematic response is verified by the experiment. In order to analyse the relationship between structural parameters and the closing behaviour intuitively, a single degree of freedom model is provided. It is shown that the mass of the control rod is the key parameter exerting important influence on the cut-off time of the valve. The cut-off time will be shortened by about $1.2 \mathrm{~ms}$ while the mass of control rod reduces $0.01 \mathrm{~kg}$.
\end{abstract}

\section{Introduction}

The mechanical quick closing valve in this paper, as a kind of stop valve with a wide application, is installed between the pressure vessel and the downstream pipe. In this way, should the pressure vessels fail, the valve can protect the equipment connected with downstream pipe from the impact flow. Shortening the cut-off time will improve the protection capability of the valve significantly. And establishing dynamic model to describe the closing behaviour is precondition for the structural optimization.

A multi-body dynamic model of the mechanical quick closing valve is established based on Lagrange equation and contact phenomena modelling method [1-4] in this paper. The effect of the structural parameters on the closing behaviour of the valve in working situation is studied. Then a optimization method of the valve is provided based on the theoretical and experimental result.

\section{Multi-body dynamic modelling of the quick closing valve}

As shown in Figure 1, the quick closing valve is actuated by a compressed spring. The control rod is linked to pressure vessel by means of a sliding transmission structure. Under normal circumstances, the connecting shaft and control rod provide braced force to keep the valve opening. When the pressure vessel fails, the control rod will be not supported anymore. Then the guiding shaft will slip down immediately because of the force of the compressed spring. So the upstream and downstream pipes will be cut off by the deformed rubber sealing element.

\footnotetext{
${ }^{\mathrm{a}}$ Corresponding author : wang13o@163.com
} 


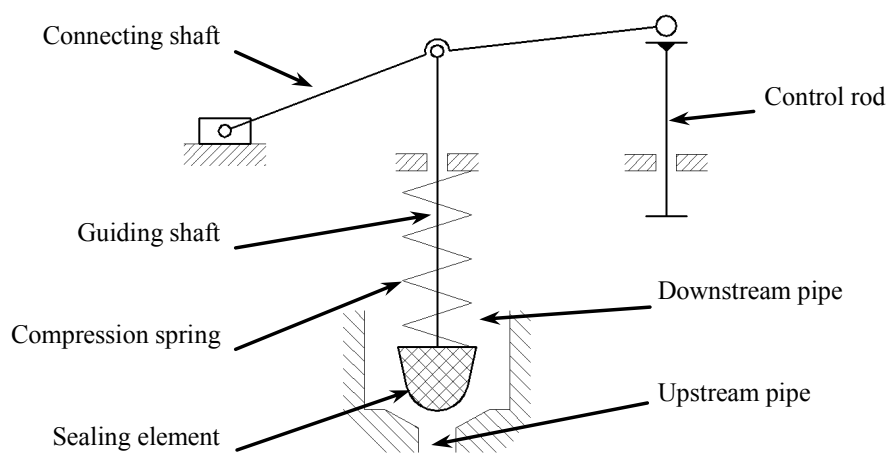

Figure 1. Structure diagram of the quick closing valve

A multi-degree of freedom dynamic model is established to quantitative describe the closing behaviour in this section. The following assumptions are applied:

a. The buffering action caused by the rubber sealing element is not considered. Because its effect on the cut-off time of the valve is slightly according to the experimental result.

b. Every component of the system is regarded as rigid.

Based on the assumptions, the kinematic response of the valve during closing process can be described by using Lagrange equation:

$$
\left\{\begin{array}{c}
\frac{d}{d t}\left(\frac{\partial E}{\partial \dot{q}_{j}}\right)-\frac{\partial E}{\partial q_{j}}+\sum_{i=1}^{n} \frac{\partial \Phi_{i}}{\partial q_{j}} \lambda_{i}=F_{i}(i, j=1 \sim 18) \\
\Phi_{i}=0
\end{array}\right.
$$

$q_{j}$-- generalized coordinate of the component barycentre (including the translation and rotation of the connecting shaft, guiding shaft and control rod)

$E$-- kinetic energy of the system

$\Phi_{j}$-- constraint equation of generalized coordinate, obtained from the geometric structure

$F_{j}$-- generalized force striking the direction of generalized coordinate

$\lambda_{j}$-- Lagrange multiplier

Once the valve switch activates, every component is in state of dry friction without lubricant. So it is significant to calculate the contact force correctly. The impact force equation applied in this paper is as follows $[5,6]$ :

$F_{n}$-- normal contact force

$$
F_{n}=K \sigma^{e}+C v_{n}
$$

$K$-- Hertz contact stiffness $\left(K=10^{8} \mathrm{~N} / \mathrm{m}\right)$

$\sigma$-- penetration depth of the contact point

$e$-- exponent of the contact force $(e=2.2)$

$C$-- damping factor $\left(C=10^{4} \mathrm{~N} \cdot \mathrm{s} / \mathrm{m}\right)$

$v_{n}$-- normal velocity of the contact pair

The nonlinear coulomb friction equation:

$$
\mu\left(v_{\tau}\right)=\left\{\begin{array}{l}
-\operatorname{sign}\left(v_{\tau}\right) \cdot \mu_{d},\left|v_{\tau}\right| \geq v_{d} \\
-\operatorname{step}\left(\left|v_{\tau}\right|, v_{d}, \mu_{d}, v_{s}, \mu_{s}\right) \cdot \operatorname{sign}\left(v_{\tau}\right), v_{s}<\left|v_{\tau}\right|<v_{d} \\
\operatorname{step}\left(v_{\tau},-v_{s}, \mu_{s}, v_{s},-\mu_{s}\right),-v_{s} \leq v_{\tau} \leq v_{s}
\end{array}\right.
$$

$v_{\tau}$-- slip velocity of the contact pair

$v_{s}$-- stiction transition slip velocity $\left(v_{s}=0.01 \mathrm{~m} / \mathrm{s}\right)$ 
$v_{d}$-- dynamic transition slip velocity $\left(v_{d}=0.1 \mathrm{~m} / \mathrm{s}\right)$

$\mu_{s}$-- static friction coefficient $\left(\mu_{s}=0.7\right)$

$\mu_{d}$-- dynamic friction coefficient $\left(\mu_{d}=0.45 \sim 0.55\right)$

The equation step is a cubic polynomial interpolating function that provides the relationship between friction coefficient and slip velocity. It is as follows with given points $\left(x_{0}, y_{0}\right)$ and $\left(x_{1}, y_{1}\right)$.

$$
\text { step }\left(x, x_{0}, y_{0}, x_{1}, y_{1}\right)=y_{0}-\left(y_{0}-y_{1}\right) \cdot\left(\frac{x-x_{0}}{x_{1}-x_{0}}\right)^{2} \cdot\left(3-2 \frac{x-x_{0}}{x_{1}-x_{0}}\right), x_{0}<x<x_{1}
$$

The solution of the dynamic model composed by Equation (1) (4) can be obtained by software conveniently. The main parameters for the model are given in Table 1.

Table 1. Table of the main parameters for the model

\begin{tabular}{|l|l|l|}
\hline No & Name & Value \\
\hline 1 & Mass of the guiding shaft / kg & 0.024 \\
\hline 2 & Mass of the connecting shaft / kg & 0.077 \\
\hline 3 & Mass of the control rod / kg & 0.093 \\
\hline 4 & Stiffness of the spring / N/m & $6.2 \times 10^{3}$ \\
\hline 5 & Preload of the spring / N & 148.8 \\
\hline 6 & Hertz contact stiffness / N/m & $10^{8}$ \\
\hline 7 & Exponent of the contact force & 2.2 \\
\hline 8 & Damping factor / N·s/m & 104 \\
\hline 9 & Stiction transition slip velocity / m/s & 0.01 \\
\hline 10 & Dynamic transition slip velocity / m/s & 0.1 \\
\hline 11 & Static friction coefficient & 0.7 \\
\hline 12 & Dynamic friction coefficient & $0.45 ~ 0.55$ \\
\hline
\end{tabular}

In order to verify the theoretical result, the experimental data of guiding shaft falling displacement and velocity during the closing process are obtained by applying high-speed photography. The comparative schemes of them are given by Figure 2. 


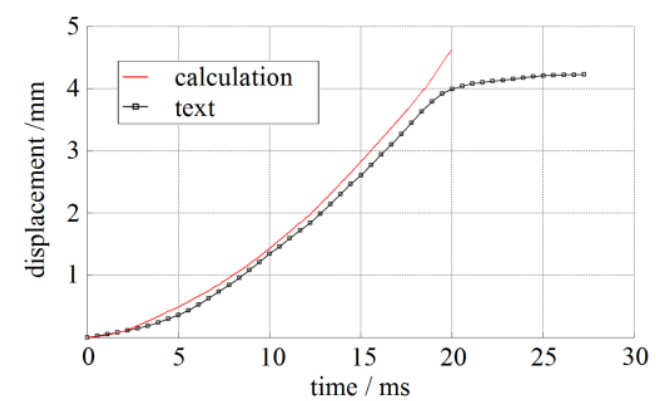

(a)

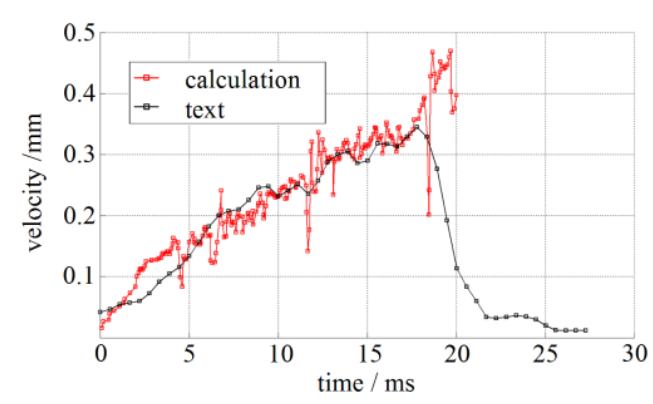

(b)

Figure 2. Theoretical and experimental result of the falling displacement (a) and velocity (b)

According to the experimental data, the valve will be closed when the falling displacement of guiding shaft reaches $4 \mathrm{~mm}$. The theoretical result of the cut-off time is $18.16 \mathrm{~ms}$, differs by $9.7 \%$ from the experimental data $(20.11 \mathrm{~ms})$. It is because the buffering action caused by the rubber sealing element is not considered. Therefore the model cannot describe the retarded motion of the guiding shaft by the end of the closing operation. However the theoretical model is accurate enough, especially in the accelerated motion phase.

\section{Structural optimization and experiment}

\subsection{Identification of the key structural parameters}

According to dynamic model in the previous section, the kinematic response of the valve during closing process is available. However it is not intuitive to analyse the effect of structural parameters on cut-off time. So a single degree of freedom model is established to describe the relationship between structural parameters and the closing behaviour.

The model is simplified further based on two additional assumptions compared to section 2.2:

c. All joints are considered to be ideal constraints. So the system is simplified as a rod mechanism with only one downward degree of freedom of the guiding shaft.

d. The contact forces, includes normal contact force and tangent friction force, are ignored.

Based on the above additional assumptions and equation (1), a concise equation of generalized coordinate and generalized force can be obtained by introducing equivalent moment of inertia $J_{e}$ :

$$
J_{e} \ddot{q}^{\prime}+\frac{1}{2} \frac{d J_{e}}{d q^{\prime}} \dot{q}^{\prime 2}=F_{k}-F_{r} \frac{\dot{y}_{r}}{\dot{q}^{\prime}}
$$

$q^{\prime}$-- generalized coordinate of the guiding shaft

$q^{\prime}$-- equivalent moment of inertia of the system

$F_{k}$-- force of the compressed spring

$F_{r}, y_{r}$-- inertial force and displacement of the control rod

The equation shows that $F_{k}$ and $F_{r}$ are the key factors of the closing operation. On the premise of maintaining the geometric parameters, the cut-off time can be shortened by adjusting the spring stiffness and the mass of the control rod. However increasing stiffness will make the spring larger and also increase the load of other structure. So the discussion will be focused on the effect of control rod mass. 


\subsection{The effect of control rod mass on the cut-off time}

In practice, the mass of control rod is about $0.093 \mathrm{~kg}$. Three kinds of light control rod are designed by applying light material and hollow structure. The numerical solution of the dynamic model composed by Equation (1) (4) and experimental data of cut-off time are obtained simultaneous (As shown in Table 2).

Table 2. Table of the cut-off time obtained by different control rod

\begin{tabular}{|c|c|c|c|c|c|}
\hline \multirow{2}{*}{ No } & \multirow{2}{*}{ Mass / kg } & \multicolumn{2}{|c|}{ Experimental result } & \multicolumn{2}{|c|}{ Numerical solution } \\
\hline & & cut-off time / ms & rate of descent & cut-off time / ms & rate of descent \\
\hline 1 & 0.093 & 20.11 & - & 18.16 & - \\
\hline 2 & 0.060 & 15.44 & $23 \%$ & 13.62 & $25 \%$ \\
\hline 3 & 0.033 & 12.58 & $37 \%$ & 10.80 & $41 \%$ \\
\hline 4 & 0.021 & 11.19 & $44 \%$ & 8.88 & $51 \%$ \\
\hline
\end{tabular}

The numerical solutions are consistent with the experimental result in every scheme. And the conclusion provided in the previous section is verified as well. The cut-off time is negatively correlated with the control rod's mass. The experimental result shows that the cut-off time will be shortened by about $1.2 \mathrm{~ms}$ while the mass of control rod reduces $0.01 \mathrm{~kg}$.

\section{Conclusions}

The multi-body dynamic model which considers the influence of normal contact and tangent friction force is provided to study the kinematic response of mechanical quick closing valve. Its numerical solution of the valve's cut-off time is verified by the high-speed photography experiment. Based on the given valve in this paper, the dynamic model can describe the closing behaviour accurately, especially in the accelerated motion phase.

A simple model is provided to analyse the effect of structural parameters qualitatively. It is shown that reducing the mass of the control rod is an effective method to shorten the cut-off time and improve the protection capability of the valve thereby. Based on the numerical solution and experimental result, there is a good linear relationship between them. The cut-off time will be shortened by about $1.2 \mathrm{~ms}$ while the mass of control rod reduces $0.01 \mathrm{~kg}$.

\section{References}

1. H. Rahnejat, P I MECH ENG C-J MEC, 214, 149 (2000)

2. G. Gilardi, I. Sharf, MECH MACH THEORY, 37, 1213 (2002)

3. S.W. Kim, Contact dynamics and force control of flexible multi-body systems (Ph.D. Dissertation, Canada, McGill University, 1999)

4. Paul, B. MECH MACH THEOR, 10, 481(1975).

5. K.E. Harald, S. Frantisek, M. Ante, PARTICUOLOGY, 8, (2010)

6. B. Shi, A study on dynamic modelling and virtual prototype of 600MW supercritical steam turbine reheat-stop-valve (Ph.D.Dissertation, China, Shanghai Jiao Tong University, 2008) 